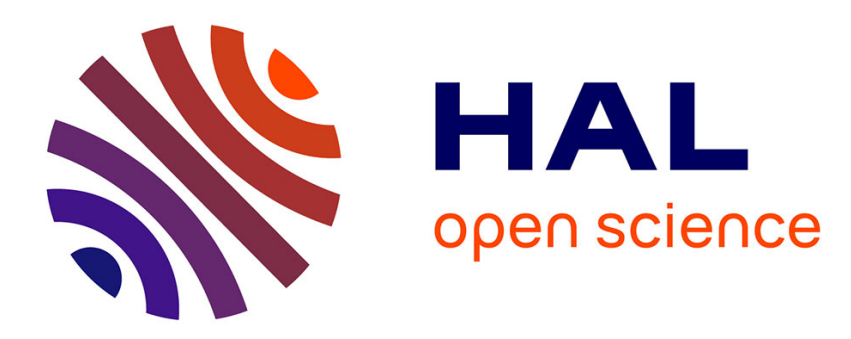

\title{
Orofacial cutaneous function in speech motor control and learning
}

Takayuki Ito

\section{To cite this version:}

Takayuki Ito. Orofacial cutaneous function in speech motor control and learning. Melissa A. Redford. The Handbook of Speech Production, Wiley Blackwell, 2015, 978-0-470-65993-9. 10.1002/9781118584156.ch12 . hal-01241995

\section{HAL Id: hal-01241995 \\ https://hal.science/hal-01241995}

Submitted on 11 Dec 2015

HAL is a multi-disciplinary open access archive for the deposit and dissemination of scientific research documents, whether they are published or not. The documents may come from teaching and research institutions in France or abroad, or from public or private research centers.
L'archive ouverte pluridisciplinaire HAL, est destinée au dépôt et à la diffusion de documents scientifiques de niveau recherche, publiés ou non, émanant des établissements d'enseignement et de recherche français ou étrangers, des laboratoires publics ou privés. 
Ito - Orofacial Curaneous Function

\section{Orofacial cutaneous function in speech motor control and learning}

\section{Takayuki Ito}


Ito - Orofacial Curaneous Function

\begin{abstract}
Somatosensory signals from facial skin can provide a rich source of sensory input. However, it is unknown yet how cutaneous input works on speech motor control and learning. This chapter introduces a kinesthetic role of orofacial cutaneous afferents in speech processing. We argue for specificity of the orofacial somatosensory system from anatomical and physiological perspectives. The contribution of cutaneous afferents to speech production is evident in neurophysiological and psychophysical findings. Somatosensory modulation associated with facial skin deformation induces a reflex for articulatory motion adjustment in speech production and also an adaptive motion change in speech motor learning. In addition, cutaneous mechanoreceptors are narrowly tuned at the skin lateral to the oral angle. An intriguing function of somatosensory inputs associated with facial skin deformation is to interact with the processing of speech perception. Taken together, orofacial cutaneous afferents play an important role in both speech production and perception.
\end{abstract}


Ito - Orofacial Curaneous Function

\section{Introduction}

Cutaneous afferents in the skin are known to be a source for kinesthetic information (sense of motion) in motor control (McCloskey 1978; Proske \& Gandevia 2009). Because the skin deforms in various ways for a given movement, cutaneous afferents associated with skin deformation related to motion can provide kinesthetic information of the corresponding movement in sensorimotor control processing. However, the prevailing view is that kinesthetic information comes largely from proprioceptors and accordingly attention to cutaneous afferents has been more limited (Proske and Gandevia 2009). Indeed most of the literature on cutaneous receptors focuses on their role in pain, thermal sensation, and touch, rather than on kinesthesia or sensation of motion (McGlone \& Reilly 2010).

Given that cutaneous mechanoreceptors are relatively dense in the facial skin as well as the skin over the hand (comparable to the skin over the trunk and limb system) (Halata \& Munger 1983; Munger \& Halata 1983), somatosensory signals arising from cutaneous afferents in the facial skin can play a crucial role in speech motor control compared with the other skeletal system, such as the limb system (Connor \& Abbs 1998; Ito \& Gomi 2007; Ito \& Ostry 2010; Johansson et al. 1988a). In addition, they are potentially valuable in understanding the kinesthetic role of cutaneous information because many orofacial structures, and notably the perioral system, lack muscle proprioceptors (Folkins \& Larson 1978; Stål et al. 1987; Stål et al. 1990) and cannot make up for this through visual input for control of articulatory motion. For these reasons, the face represents a model system for examining the kinesthetic role of 
Ito - Orofacial Curaneous Function

cutaneous afferents. Knowing the functional role of facial skin deformation can thus offer a new way of understanding orofacial somatosensory function in speech processing.

This chapter focuses on the kinesthetic role of orofacial cutaneous afferents in speech processing and how somatosensory signals arising from cutaneous afferents in the facial skin contribute to speech motor control and learning. Section 2 summarizes anatomical and physiological foundations in the facial proprioceptive system in comparison with limb proprioception and addresses the importance of cutaneous afferents in facial motor control. Section 3 describes the neural activity of orofacial cutaneous afferents in speech motion based on the physiological studies using microelectrode recording. Sections 4 and 5 describe the functional role of cutaneous afferents in speech motor control and learning in terms of kinesthetic function. Section 6 considers the contribution of the somatosensory system to the processing of speech sounds from the aspect of orofacial cutaneous function. Together these sections link concepts of orofacial cutaneous afferents and provide a basis for the kinesthetic role of orofacial cutaneous afferents in speech processing.

\section{Anatomical and physiological foundations of the orofacial somatosensory system}

The sensory organs for proprioception have been primarily investigated for the limb skeletal system. There has been limited attention directed to orofacial proprioception including cutaneous mechanoreceptors. Indeed, common understanding to date is that muscle proprioceptors (muscle spindles and tendon organs) are the main source for the sense of motion needed for motor control of the various skeletal systems. Given strong evidence of the importance of muscle spindles and tendon system for the sense of motion, 
Ito - Orofacial Curaneous Function

the following questions arise: Does the orofacial system behave in the same way as limb proprioception? Are muscle proprioceptors the main source of kinesthetic information in speech motor control? To facilitate comparison with the orofacial system, we begin addressing these questions by introducing the basic physiological function of muscle proprioceptors associated with reflex. The overall aim of this section is to describe specifics of orofacial proprioception based on the current findings.

\subsection{Orofacial muscle proprioceptors}

Muscle proprioceptors (muscle spindles and tendon organs) are sensory organs in muscles that provide the sense of motion (McCloskey 1978; Proske \& Gandevia 2009). Muscle spindles are the mechanoreceptors in muscles that detect a change of muscle contraction (or stretch). The role of muscle spindles in sensorimotor control can be seen in various reflexes. A representative example is the stretch reflex that maintains the same limb posture when the limb is suddenly flexed or extended due to external disturbance (Marsden et al. 1972). Muscle length change due to sudden stretch is coded in the discharge of muscle spindles as motor error. Since spindle afferents monosynaptically connect to motor neurons in the spinal cord, the motor error signal arising from muscle spindles directly drives compensatory activation in the motor neurons. This additional discharge in the motor neurons results in a contraction of the stretched muscle to maintain the same muscle length. Because its functional and neural characteristics have been well investigated, the stretch reflex is an effective means to assess muscle spindle function for scientific hypothesis testing or clinical diagnosis. Physiological characteristics of muscle spindles are also exemplified by the tonic vibration reflex (TVR). TVR induces 
Ito - Orofacial Curaneous Function

additional muscle contraction (increasing generated force) when vibratory stimulation is applied to a muscle or tendon. Vibratory stimulation of a muscle stimulates muscle spindles in the absence of an obvious muscle stretch.

Another representative proprioceptor is the tendon organs that connect skeletal muscle to bone. Tendon organs are known to provide information of muscle tension force in order to protect muscles from excessively heavy loads. Like muscle spindles, the reflex called the tendon reflex illustrates a kinesthetic function of tendon organs. The tendon stretch reflex is commonly elicited in clinical examinations by tapping the tendon with a rubber hammer. Interestingly, vibratory stimulation to the tendon organs causes an illusionary perceptual sensation, that is, the feeling that the stimulated muscle is being stretched (Goodwin et al. 1972). This illusionary sensation is used as a mean to investigate muscle proprioceptive function in motion (Cordo et al. 1995).

The fundamental functions of muscle proprioceptors including reflex function have been examined in the orofacial system to determine whether proprioceptive function in the orofacial system is the same as in the limb system. In the speech articulatory system, the lip, tongue and jaw are the main articulators to determine the specific vocal tract shape for the production of vowels and consonants. Here we discuss muscle proprioceptors of the lip and jaw mainly because the lip and jaw motion are always accompanied by facial skin deformation.

Lip motion is achieved by a combination of multiple muscle contractions (orbicularis oris superior and inferior, buccinators, risorius, major and minor zygomaticus, depressor anguli oris, levator labii superior and inferior, mentalis). Each muscle works separately or together for specific lip motion (O'Dwyer et al. 1981). For 
Ito - Orofacial Curaneous Function

example, orbicularis oris superior and inferior predominantly control lip protrusion and rounding. Unlike the other skeletal muscles, lip motion is the result of adding the directional forces from a combination of several muscle contractions. Hence no skeletal movement is involved in lip motion.

Several studies have attempted to assess whether lip muscles have muscle proprioceptors. Anatomical studies (Stål et al. 1987; Stål et al. 1990) showed no evidence of muscle spindles in several lip muscles: orbicularus oris, buccinators, major and minor zygomaticus. Neilson et al. (1979) approached physiologically the existence of muscle spindles by examining the stretch reflex. They stretched the lip in a variety of ways to make sudden muscle stretches and recorded electromyography from most lip muscles (orbicularis oris, major zygomaticus, levator labii inferior, depressor anguli oris, depressor labii inferioris, mentalis, and buccinator). No evidence of stretch reflex was observed, suggesting an absence of muscle spindles. Folkins and Larson (1978) examined tonic vibration reflex, that is, the other typical reflex driven by muscles spindles. When vibratory stimulation was applied to the lip, no additional force was found in measurement of the lip force using a force transducer, consistent with the absence of muscle spindles.

In addition to the lack of muscle spindles, there is no report of tendon organs in the lip muscles. Since the lip is not a system for generating skeletal motion like the limb system, one end of the muscle or its entire body does not connect to the skull or mandible bone directly. Rather, the lip muscles are intermingled with each other to make a connection (McClean \& Smith 1982). In particular, multiple lip muscles are concentrated at the corners of the mouth. These anatomical and physiological findings provide no 
Ito - Orofacial Curaneous Function

evidence for muscle proprioceptors in lip muscles, and in fact suggest their absence and the need for an alternative source of proprioceptive information for lip movement.

The jaw is a system similar to the limb system in that muscle contraction generates skeletal motion. But one difference from the limb system is that the jaw has asymmetrical requirements for force generation between opening and closing motions, whereas the limb system has approximately symmetrical requirements for flexion and extension movement. Jaw closing requires precise force control with a large force for mastication of a variety of foods, but relatively imprecise control with much less force is sufficient for jaw opening. This asymmetrical functional requirement may directly be seen in the configuration of muscle proprioceptors. Jaw closing muscles, particularly the masseter and temporalis, have rich muscle spindles, although there is relatively smaller number of spindles in lateral pterygoid (Kubota \& Masegi 1977). Moreover, the muscles spindles in the masseter are larger and more complex than in limb muscles (Eriksson et al. 1994). This might be due to the precise control needed for mastication.

Muscle spindles in the jaw closing muscles typically show the same reflexes driven by muscle proprioceptors as the limb muscles. They induce stretch reflexes called the jaw jerk reflex (Lund et al. 1983; Miles et al. 2004) and the tonic vibration reflex (Eklund \& Hagbarth 1966). These reflexes suggest that muscle spindles in the jaw closing muscles play a role in providing kinesthetic information like those in the limb system as servo control mechanisms shown in Lamarre and Lund (1975). On the other hand, spindles may not be essential source of sensory information during jaw opening because muscle spindles are rarely present in jaw-opening muscles (digastricus, mylohyoid, geniohyoid and lateral pterygoid). Lennartsson (1979) found only a few 
Ito - Orofacial Curaneous Function

muscle spindles in digastricus, but not in all muscles that were investigated in this study or in all individuals. They concluded muscle spindles in jaw opening muscles are not an essential source of sensory input. The tonic stretch reflex was also examined in the digastric muscles. The muscle response changed, depending on motor parameters such as joint torque and jaw orientation, despite the fact that there are few or no muscle spindles in these jaw opening muscles (Ostry et al. 1997). This suggests that there must be an alternative source of proprioceptive inputs other than muscle spindles for jaw opening.

It is not well studied yet about the proprioception of the tongue muscles, however it might also have different characteristics of proprioceptors from limb system that is generally known in the textbook. In the extrinsic tongue muscle (e.g. the genioglossus), proprioceptive information seems to be available from muscle spindles, which have been found (Cooper 1953). However, like the muscles of the lips, tongue extrinsic muscles do not show any evidence of a stretch reflex (Neilson et al. 1979), suggesting that muscle spindles in the tongue may not work in the same way as in the limb systems. Different from the lip muscles, tongue extrinsic muscles are connected to the mandibular symphysis by the short tendon (Takano \& Honda 2007), although its sensory function is not known yet.

To summarize, current anatomical and physiological evidence shows that the orofacial system is not the same as the limb system with respect to sensing motion. In particular, a paucity of muscle proprioceptors in perioral muscles strongly suggests the contribution of some other source of proprioceptive inputs, such as cutaneous afferents.

\subsection{Orofacial skin receptors}


Ito - Orofacial Curaneous Function

Cutaneous mechanoreceptors are relatively densely innervated in facial skin compared to skin over other parts of the body (Halata \& Munger 1983; Munger \& Halata 1983), and the corners of the mouth is the most densely innervated area in the face (Johansson et al. 1988a; Nordin \& Hagbarth 1989). Like the skin on the palm of one's hand, the oral and perioral regions have outstanding tactile spatial acuity as determined by two-point discrimination task (Weinstein 1968). In fact, there is an anatomical difference between facial skin and the skin over other parts of the body. In general knowledge of skin receptors, there are several types of mechanoreceptor--Ruffini corpuscles, Meissner corpuscles, Merkes disk receptors, and Pacini corpuscles, hair follicle fibers, and free nerve endings. Interestingly, the Pacini corpuscles, which are well represented in the fingertips and the palm of the hand where they are responsible for detection of high-frequency vibrations, are absent in the facial skin. In microelectrode recording of cutaneous afferents of peri- and intra-oral tissue, no afferents show response properties similar to typical Pacinian-corpuscle afferents (Johansson et al. 1988b). This is supported by physiological tests using vibro-tactile stimulation showing that Paciniantype frequency sensitivity is absent in the face (Barlow 1987). However, it is not clear yet how the lack of Pacini corpuscles in facial skin affects facial skin sensory process including the sense of motion. Whereas there is anatomical difference from the skin over other parts of the body, facial cutaneous afferents are similar to the afferent types described in human hand in terms of rate of adaptation to constant or static stimulation (Trulsson \& Johansson 2002). Those consist of three types of afferents: Fast adapting and Slowly adapting (Type I and Type II) afferents. In the facial skin and the transitional 
Ito - Orofacial Curaneous Function

zone of the lip, a majority of the afferents have slowly adapting property (Johansson et al. 1988b).

Sensory inputs arising from facial cutaneous mechanoreceptors are conveyed through the trigeminal nerve. The trigeminal nerve has three major branches: the ophthalmic nerve, the maxillary (or infraorbital) nerve, and the mandibular nerve. These branches innervate separate facial areas. Roughly, the ophthalmic nerve is for the upper part of the face: the scalp, forehead, upper eyelid and nose. The maxillary nerves are for the middle part of the face: cheek, lower eyelid, and upper lip. The mandibular nerve is for the lower part of the face: the lower lip and jaw. The ophthalmic and maxillary nerves are purely sensory. The mandibular nerve has both sensory and motor functions. Since the maxillary nerve and the mandibular nerve are mostly involved in the sense of speech motion, only the cutaneous afferents arising from these two nerves are discussed in this chapter.

The mandibular nerve controls motor function in the jaw muscles. The fact that this one nerve has both motor and sensory function is similar to the nerves that innervate limb muscles. The similarity between jaw closing muscles and limb muscles is reflected in the fact that the stretch reflex, which is transmitted via monosynaptic loop in the skeletal muscles. As noted above, though, this reflex is evident only in the jaw closing muscles.

Different from the jaw closing muscles, two physically separate nerves: the facial nerve and trigeminal nerve innervate the lip region for motor function and for sensory function respectively. These two nerves originate from separate nuclei in the spinal cord, suggesting a lack of monosynaptic connection from sensory afferents to motor neurons. 
The lack of monosynaptic connection is also consistent with the lack of spindle-like receptors or function in the perioral system.

Orofacial cutaneous afferents are polysynaptically connected to the facial motor system in the subcortical level. A typical example is perioral reflex seen in one of the lip muscles (orbicularis oris). Brief tapping on the lip is a common method to evoke the perioral reflex (Bratzlavsky 1979). Stretching the lip lateral to the oral angle also induces the reflex in the lip muscles (Ito \& Gomi 2007; Larson et al. 1978; McClean \& Smith 1982). The latency of the perioral reflex [approximately $16 \mathrm{~ms}$ : McClean and Clay (1994), Smith et al. (1985a)] is approximately twice as long as the jaw jerk reflex [approximately $8 \mathrm{~ms}$, Murray and Klineberg (1984)]. Given that the jaw jerk reflex is driven via monosynaptic loop, the approximately doubled perioral latency despite almost the same travel distance indicates that the perioral reflex spends more time due to going through multiple neural connections.

The function of the perioral reflex in orofacial motor control is still controversial. The amplitude of the perioral reflex is slightly suppressed prior to speech production (McClean \& Clay 1994), but not during sustained phonation (Smith et al. 1985a). The effect of cutaneous afferents arising from the lip (or sensory nerve of the lip) is not limited only to the orbicularis oris. Air-jet stimulation of the lip or electrical stimulation to orofacial tactile nerves induces inhibitory responses in jaw closing muscles (Di Francesco et al. 1986; Okdeh et al. 1999). Stretching the facial skin lateral to the oral angle also induces a similar inhibitory response in the jaw closing muscle (Ito \& Ostry 2010). This indicates a neural connection of facial cutaneous afferents to the motor system of two main articulators in the subcortical level. 


\section{Cutaneous activation in facial motion}

Lip and jaw motion is normally accompanied by facial skin deformation, which occurs broadly in the overall lower facial area in several tasks: lip protrusion, chewing, and speaking (Connor \& Abbs 1998). The range of skin strain in response to lower lip motion is greater than the threshold of skin strain in cutaneous mechanoreceptors [a minimal strain sensitivity of 0.0125 is reported in Edin (1992)]. Facial skin deformation during various movement tasks was of sufficient magnitude to elicit discharge from cutaneous mechanoreceptors. In addition, displacement of the lower lip can be estimated from the amount of skin stretch in the lower facial area. Displacement of facial skin deformation during speech motion is also effective to estimate corresponding tongue motion and speech acoustics (Vatikiotis-Bateson et al. 1999; Yehia et al. 1998).

Actual activation of facial cutaneous mechanoreceptors during motion has been observed in microelectrode recording of facial sensory nerve. Cutaneous mechanoreceptive afferents in the infraorbital nerve, which innervate the middle part of the face, discharge due to the deformation of the facial skin associated with various phases of voluntary lip and jaw motion, including speaking motions (Johansson et al. 1988a; Nordin \& Thomander 1989). In speech tasks, cutaneous afferents show biphasic activity prior to the production of the explosive sound /p/ or /b/ (Johansson et al. 1988a). The first phase of the biphasic activation corresponds to the lip closing motion in a bilabial articulation. The second phase relates to the air pressure build up for explosive sounds. This activation has been observed in the cutaneous afferents that have their receptive fields close to the corners of the mouth. Cutaneous mechanoreceptors from the 
corners of the mouth also discharge during lip protrusion in non-speech tasks (Nordin \& Thomander 1989). In chewing, discharge of cutaneous mechanoreceptors shows a biphasic discharge per one jaw cycle; the equivalent of a single jaw opening and closing motion (Johansson et al. 1988a; Nordin \& Thomander 1989). Externally applied skin stretch, in the absence of actual speech articulator motion, also induces similar cutaneous activation (Nordin \& Hagbarth 1989; Nordin \& Thomander 1989). When the skin above the upper lip is stretched in the lateral direction by pulling an adhesive tape attached outside the receptive field, a dynamic on and off discharge is clearly induced. Static deformation induces less discharge.

Detailed kinesthetic characteristics of cutaneous discharge pattern associated with motion-related skin deformation have been examined in limb studies. Finger skeletal motion is relatively easier to map into nerve activation associated with skin deformation than facial motion. Cutaneous mechanoreceptors in dorsal skin of the hand discharge due to flexion and extension of the finger (Edin \& Abbs 1991). Directional responses to these joint movements have been seen in a response of the cutaneous mechanoreceptors that have characteristics of slowly adapting to continuous stimulation. Flexion motion induces greater activity in slowly adapting mechanoreceptors than extension motion. Velocity sensitivity has also been examined in the finger extensor muscles (extensor digitorum). In a recording of slowly adapting mechanoreceptors and muscle spindles, discharge pattern of both types of receptors was proportional to velocity of ramp flexion movements (Grill \& Hallett 1995). This finding is consistent even with a wider area of skin deformation during motion. The response of slowly adapting cutaneous afferents in the thigh reveals both dynamic and static aspects of knee joint movements (Edin 2001). The same group of 
slowly adapting units also discharge due to manually applied skin stretch. These results suggest peripheral cutaneous activation pattern in responses to motion-related skin deformation effectively encodes direction and velocity information.

In addition to peripheral neural responses, cortical responses associated with motion-related skin deformation have also been studied in direct cortical recording in an awake monkey. Skin deformation in an arm movement task generated tactile activity in primary somatosensory cortex (Cohen et al. 1994; Prud'homme et al. 1994). This indicates that skin strain due to motion induces the discharge of cutaneous afferents that is similar to other stimulations to the skin (painful, thermal and touch stimulations). Activity in primary somatosensory cortex supports the idea that cutaneous afferents play a kinesthetic role in motor control.

Further quantitative analysis using a different type of cutaneous stimulation to facial skin have provided more understanding of how tactile information is decoded during cortical processing. Brush stimuli applied to the facial and finger hairy skins induce direction-dependent activation patterns in microelectrode recording of cutaneous afferents (Edin et al. 1995). Brush stimulation in the same direction shows a consistent spatial pattern of cutaneous activation and the stimulation in another direction shows a different consistent pattern. However, a consistent pattern of activation may not be used to detect motion information such as direction and velocity, since it is necessary to process the activation in the temporal domain in order to obtain velocity information, but not in the special domain as observed in here. Instead of special pattern consistency, it is likely that velocity and direction information from a moving tactile stimulus is coded by the mean firing rate in the population of excited mechanoreceptors (Essick \& Edin 1995). 
Ito - Orofacial Curaneous Function

Facial cutaneous mechanoreceptors respond to motion of the skin the same way as other cutaneous mechanoreceptors respond to motion in the finger and arm. Since the activation patterns of cutaneous afferents register dynamical characteristics of movement, the cutaneous mechanoreceptors can code the kinesthetic information needed for motor control. The skin at the corners of the mouth may be especially important to motor control because cutaneous mechanoreceptors are the most densely innervated there and show activation in response to movement of the speech articulators. This idea is further discussed in the following section.

\section{Cutaneous contribution in speech motor control}

The kinesthetic role of cutaneous mechanoreceptors in the speech motor system has been assessed in a study that investigated the compensatory speech adjustments (Ito \& Gomi 2007). The quick compensatory response examined was that of the upper lip motion during the production of the bilabial fricative consonant $/ \phi /$. Precise lip constriction is required in bilabial fricative consonants to achieve the production of fricative noise. When jaw position is unexpectedly shifted downward by an external force disturbing lip constriction, the upper lip quickly compensates by an additional downward shift in order to achieve an intact labial aperture (Gomi et al. 2002). This quick compensatory motion is driven by two mechanisms in sequence. A mechanical component due to muscle linkage (Gomi et al. 2002; Ito et al. 2004) works for the initial phase and a transcortical reflex works for the following phase (Ito et al. 2005). While the mechanical component due to muscle linkage is planned in advance for the motion, the transcortical reflex is driven by sensory error signals due to the sudden position change of 
the jaw (or the lower lip). Although muscle spindles are rich in the jaw closing muscles, if orofacial cutaneous mechanoreceptors contribute to providing motion information for the jaw together with muscle spindles, the compensatory reflex should be induced by orofacial skin deformation associated with the jaw motion in the absence of actual jaw position change. To test this hypothesis, Ito and Gomi (2007) disrupted participants' production of the bilabial fricative by pulling the skin lateral to the oral angle downward while jaw position was held constant. As expected, the compensatory reflex was induced. The compensatory reflex of the upper lip in response to facial skin stretch suggests that cutaneous mechanoreceptors can provide sensory error signals that are associated with jaw motion. In this way, we find that orofacial cutaneous afferents contribute directly to speech motor control.

Although deformation of the facial skin is more or less distributed in the broad area of the lower face during speech motion (Connor and Abbs 1998), cutaneous mechanoreceptors in the skin lateral to the oral angle might be predominantly responsible for the detection of speech articulatory motion. This idea has already been suggested in the previously-mentioned physiological observation in neural recording that cutaneous mechanoreceptors lateral to the oral angle are activated in jaw motion (Johansson et al. 1988a) and the area around the oral angle is the most densely innervated (Johansson et al. 1988b, Nordin and Hagarberth 1989). To test this idea, facial skin stretch perturbations were applied at several sites other than lateral to the oral angle and examined which area of the facial skin is predominantly involved in lip compensatory reflex (Ito \& Gomi 2007). There was no evidence for induction of the compensatory reflex in the facial skin except that lateral to the oral angles. This indicates that the skin stretch lateral to the oral 
Ito - Orofacial Curaneous Function

angle plays a predominant role in detecting jaw motion. The facial skin stimulation to the same area also modifies the lip motion over the course of training and the perception of speech sounds, both of which are discussed in the following sections. Taken together these suggest the mechanoreceptors may be narrowly tuned in the facial skin lateral to the oral angle to detect lip and jaw articulatory motion.

Kinesthetic contribution of cutaneous mechanoreceptors is also apparent in limb studies. These studies have examined how the stimulation associated with skin deformation induces sensations of limb location and motion. Skin stretch is carefully applied without producing any position change in the manipulated limb. In the index finger, when skin strain patterns that are usually associated with finger flexion or extension were applied in the absence of passive position change, the movement-related skin strains were correctly perceived as flexion or extension motion depending on the pattern of skin stretch even when both skin and deeper tissues were anesthetized (Edin \& Johansson 1995). Other examples of the skin stretch effect were seen in movement illusions due to tendon vibratory stimulation. When vibratory stimulation are applied at the wrist, where there are tendon organs for finger muscles, without producing actual finger flexion we nonetheless feel the sensation that the finger is gradually being flexed. When the same tendon vibration is applied in combination with a stretch of finger skin, we feel a greater sensation of motion than the case of tendon vibration alone (Collins et al. 2000). This illusionary effect is not limited to the finger but is also observed in the forearm and leg (Collins et al. 2005). These results suggest that stretching the skin can cause motion-related sensation and that cutaneous mechanoreceptors provide the information of motion. 
Ito - Orofacial Curaneous Function

Skin stretch stimulation is presumably limited to activation of cutaneous mechanoreceptors, particularly in the facial system. Supportive evidences have been examined by observing the effect on the jaw muscle spindles. Jaw muscle spindles are known to be sensitive to muscle length change because the jaw-jerk reflex has been readily induced using percutaneous indentation as small as $1 \mathrm{~mm}$ to the masseter (Smith et al. 1985b). There is however no excitatory reflex when the percutaneous stimulus is delivered in a motion parallel to the skin surface on the masseter exactly above the location where the jaw-jerk reflex can be induced by indentation. Similarly the skin stretch lateral to the oral angle does not show any indication of the jaw-jerk reflex; rather it shows an inhibitory reflex that is generally induced by facial cutaneous stimulation, such as by air-puff or electrical stimulation (Ito \& Ostry 2010). This suggests skin stretch stimulation affects only cutaneous mechanoreceptors and not muscle spindle activation.

Electrical stimulation is an alternative method for stimulating cutaneous mechanoreceptors. Electrical stimulation to spindle afferent nerves produces an illusory sensation of movement and distorts their position in the absence of overt movement (Gandevia 1985). Likewise, electrical stimulation to the cutaneous sensory afferents induces motion illusions (Collins \& Prochazka 1996). However the sensation of motion due to electrical stimulation is less than that of stretching the skin. Thus, stretching the skin may be a more effective tool for investigating the kinesthetic role of cutaneous mechanoreceptors than electrical stimulation.

In addition to studies on skin stretching, the contribution of facial cutaneous mechanoreceptors in speech motor control is also apparent in studies that deliver mechanical perturbations to the lip. Given that lip muscles lack muscle spindles, if 
Ito - Orofacial Curaneous Function

motion error information is transmitted then it must be transmitted through orofacial cutaneous afferents. In Gracco and Abbs (1985), mechanical perturbation was applied to the lower lip during the production of bilabial explosive sounds $/ \mathrm{p} /$ or $/ \mathrm{b} /$, producing a sudden depression of the lower lip just before lip closure. This sudden depression of the lower lip was immediately compensated by the additional downward movement of the upper lip. The compensatory movement resulted in intact lip closure and accurate production of the plosive sound.

Although cutaneous afferents presumably play a predominant role in detecting motor error due to mechanical lip perturbation, the contribution of muscle spindle in the jaw closing muscles cannot be ruled out because the jaw is also involved in producing lower lip position. To rule out such contributions, Shaiman and Gracco (2002) conducted a study in which they perturbed the upper lip during production of plosive / $\mathrm{p} /$ and labiodental fricative $/ f /$. The perturbation to the upper lip induced compensatory motion in both upper and lower lip for the production of $/ \mathrm{p} /$, but no compensatory motion for the production of /f/ because the upper lip is not involved in its production. Since upper lip motion, unlike lower lip motion, is independent of jaw motion, cutaneous mechanoreceptors are the only available sensory organs for detecting motor errors. Given the evidence of task dependent compensatory motion, the conclusion is that somatosensory information associated with skin deformation contributes to the adjustment of speech articulatory motion in multiarticulatory coordination.

\section{Orofacial cutaneous contribution to speech learning}


Just like acoustic information, somatosensory information is important to speech motor learning. Tremblay et al. (2003) showed motor errors due to external force are corrected over the course of training independent of speech sounds. For the production of a high-low vowel sequence $/ \mathrm{i}-\mathrm{a} /$, the jaw trajectory shows an almost straight line in normal production. Tremblay et al. applied a velocity-dependent perturbation force perpendicular to the movement direction with amplitude proportional to the velocity of motion during production of the $/ \mathrm{i}-\mathrm{a} /$ sequence in a speech motor learning task. At the beginning of training, the jaw trajectory followed a curved line in the protrusion direction because the perturbation force peaked at the mid-point of jaw opening. After a number of repetitions with the jaw perturbation, the jaw trajectory eventually returned to the original approximately straight line. Since the produced vowel sounds did not change over the course of the adaptive motion change, the results suggest that motor error correction works independently of acoustic output. This conclusion is further supported by work with profoundly deaf patients, who show the same adaptive change in motion even when their cochlear implants were off (Nasir \& Ostry 2009). Together these studies suggest that somatosensory goals are set independently of acoustic goals to some extent.

Some individuals even seem to rely more heavily on somatosensory than auditory feedback during speech production (Lametti et al. 2012). When the jaw perturbation mentioned above is applied together with altered auditory feedback, individuals adapt to either just to one or both sensory modulations. Interestingly some individuals preferentially adjusted to somatosensory modulation alone, ignoring audition.

Whereas jaw perturbation studies demonstrate the crucial role of somatosensory function in speech motor learning, they are unable to dissociate the contribution of 
Ito - Orofacial Curaneous Function

cutaneous from proprioceptive receptors because jaw motion, uniquely in the orofacial system, also relies on the contribution of muscle proprioceptors. Given that muscle and joint receptors are absent in perioral muscles, the face represents a model system for examining the role of cutaneous afferents in motor learning.

As might be expected, deforming the facial skin over the course of training induces motor adaptive change in speech production. Ito and Ostry (2010) applied gentle facial skin stretch in a regular adaptation paradigm using a speech production task. For the production of /w/ in "wood", in which the lips are required to protrude more than for the production of the following / $\mathrm{u} /$ vowel, robotic devices gently stretched the facial skin lateral to the oral angle and backwards in the periods just before the onset of the target speech gesture. When the amplitude of upper lip protrusion was tracked over the course of training, the findings were that upper lip protrusion was gradually increased over the course of the training. This change was maintained as an aftereffect in the trials that followed facial skin deformation. As with the other speech motor learning studies (Nasir \& Ostry 2008; Tremblay et al. 2008; Tremblay et al. 2003), the somatosensory learning process did not affect the acoustic output.

Progressively increasing lip protrusion in response to skin stretch is in contrast to the studies of motor learning in that used jaw motion perturbation (Nasir \& Ostry 2008; Tremblay et al. 2008; Tremblay et al. 2003) in that facial skin stretch was applied in a direction opposite to the upcoming movement. It could be that the opposing stimulus resulted in sensory input that led the nervous system to underestimate lip position. Consequently, the actual motion may have been consistently evaluated as smaller than 
Ito - Orofacial Curaneous Function

the intended one, and motor commands may have been updated to progressively to yield larger movement.

Separate from the adaptive change of lip protrusion, the Ito and Ostry (2010) study also showed a compensatory response due to backward skin stretch. In order to overcome a backward skin stretch, the lip has to be driven with greater force than usual to attain the same lip protrusion target. Since the skin stretch perturbation was removed before the production of the target $/ \mathrm{w} /$, the greater compensatory force simply resulted in greater lip protrusion than usual. This compensatory lip protrusion was evident at the beginning and end of training. In the first trial of training, the amplitude of lip protrusion was suddenly increased by some amount. This same amplitude difference was also observed when the skin stretch was removed in the first trial after training, and the gradual adaptive increase over the training remained. The findings of initial change and afteraffects suggests that the online compensatory process might be driven separately in any adaptation process.

Ito and Ostry (2010) also assessed the generalization of learning using the facial skin stretch paradigm to determine whether the pattern of adaptation acquired in the context of the training task transferred to other speech movements that involved lip motion of different amplitudes. The consonant $/ \mathrm{h} /$ was used for the transfer task as it involves a different pattern of lip protrusion than the production of $/ w /$. In this test, training was carried out using the same production of $/ \mathrm{w} /$ in "wood" as previously. A similar gradual change in the production of /w/ was observed over the course of the training. However, when the transfer task /h/ in "hood" was produced immediately after the training (in the absence of skin stretch perturbation), only a limited amplitude of the 
Ito - Orofacial Curaneous Function

trained lip protrusion was transferred. This is consistent with the findings from a jaw perturbation speech motor learning study (Tremblay et al. 2008).

Results from these studies indicate that somatosensory inputs arising from facial skin deformation and jaw perturbation contribute to speech motor learning. The findings document the involvement of cutaneous afferent information in motor learning in the orofacial system. The progressive increase due to somatosensory error suggests that the nervous system produces motor commands with the expectation that sensory input correctly signals kinematic error.

\section{Somatosensory function in speech perception}

Speech perception is not the simple processing of auditory signals, but a complicated process involving the integration of multiple sensory inputs. For example, visual information from a speaker's face can enhance or interfere with accurate auditory perception. In a noisy environment, looking at a talker's face greatly improves the perception of speech sounds (Sumby \& Pollack 1954). In the McGurk effect (McGurk \& MacDonald 1976), when the auditory component of one sound (e.g. /ba/) is paired with the visual component of another sound (e.g. /ga/), a third sound can be perceived (e.g. /da/). Besides visual inputs, interactions between auditory and somatosensory information may be relevant to the neural processing of speech, since speech processes and certainly speech production involve auditory information as well as inputs that arise from the muscles and tissues of the vocal tract.

This idea is addressed from a somatosensory aspect using facial skin stretch. When the facial skin is stretched while people listen to words in the absence of any 
volitional speech motion, it alters the sounds they hear (Ito et al. 2009). For example, in Ito et al., listeners made a forced-choice identification of the words "head" or "had" when one of 10 possibilities on a continuum between "head" and "had" was presented. During this identification task, the skin lateral to the oral angles was pulled either upward, downward, or backward. Systematic perceptual variation was induced, which depended on the direction of skin stretch. When the skin was pulled upward, the sound was identified as "head" more than "had". This tendency was reversed when the skin was pulled downward. There was no evidence for perceptual change when the skin was pulled backward. Considering that difference of articulatory motion between "head" and "had" is characterized by the vertical position of the jaw and tongue, the perception of speech sounds is altered by speech-like patterns of skin stretch in a manner that reflects the way in which auditory and somatosensory effects are linked in speech production. Somatosensory inputs affect the neural processing of speech sounds and show the involvement of the somatosensory system in the perceptual processing of speech.

A reverse effect is also true in that speech sounds can alter the perception of facial somatosensory inputs associated with skin deformation (Ito \& Ostry 2012). Ito et al. investigated whether speech influences the perception of amplitude between two sequential facial skin deformations that would normally accompany speech production. The skin stretch was applied at the lateral to the oral angle in upward direction. The auditory stimuli "head" or "had" were timed to coincide with the skin stretch. The main manipulation was the order in which the speech sounds were presented for the two sequential stretches. In one condition, the word "head" was presented with the first skin stretch, and the word "had" was presented with the second skin stretch. In the other 
condition, the opposite order was used. Somatosensory judgment was that the force with the skin was stretched during the sound "had" was greater even though the actual force was the same for both speech stimuli. Moreover, somatosensory judgments were not affected when the skin deformation was delivered to the forearm or palm or when the facial skin deformation accompanied nonspeech sounds. This suggests that the modulation of orofacial somatosensory processing by auditory inputs is specific to speech and likewise to facial skin deformation. The perceptual modulation in conjunction with speech sounds shows that speech sounds specifically affect neural processing in the facial somatosensory system and suggest the involvement of the somatosensory system in both the production and perceptual processing of speech.

This might be also examined in the interaction between speech perception and overt speech production although somatosensory and motor function are equally involved in the case of actual speech production. Similar to the McGurk effect in which incongruent visual stimulation modifies the perception of a speech sound, our own motion itself can affect the perception of speech sounds (Sams et al. 2005). In this study, while listening to one series of sounds (e.g. " $p a$ "), the speaking motion associated with an incongruent sound (e.g. " $k a$ ") was produced silently. The presented sound was mostly perceived as a third sound ("ta") or the articulated sound (e.g. " $k a$ "). Although the amplitude of the effect induced by silently speaking is smaller than that produced through visual feedback, sensorimotor process in speech production clearly interact with the perception of speech sounds. As an opposite effect, somatosensation during speech motion is also changed as a consequence of altered auditory feedback. When the voice that you are speaking was amplified by external manipulation during a sustained voiced 
Ito - Orofacial Curaneous Function

sound, /u/, participants reported a throbbing sensation over the lip and laryngeal regions (Champoux et al. 2011).

Apart from the kinesthetic role of orofacial cutaneous afferents, the tactile sense from the other body part also contributes to the perception of speech sounds by detecting information movement associated with speaking. Tadoma method has been developed for deaf-blind individuals as a tactile communication method [See Reed et al. (1985) for review]. In Tadoma, a hand is placed on the talker's face in order to monitor actions associated with speech production. Performance is roughly equivalent to that of normal listening in noise. In addition, perceptual modulation like the McGurk effect can be observed if the information detected by the hands is incongruent with that which is detected by audition (Fowler \& Dekle 1991).

A passive tactile sense might aid in perceiving speech sounds in daily-life situations. For example, some speech sounds like /p/ produce tiny bursts of aspiration. Gick and Derrick (2009) showed that when listeners feel a puff of air, delivered to the hand or neck while hearing either aspirated $(/ \mathrm{pa} /$ and $/ \mathrm{ta} /)$ or unaspirated sounds $(/ \mathrm{ba} /$ and /da/), syllables heard simultaneously with air puffs were more likely to be heard as aspirated than as unaspirated sounds.

The contribution of tactile sensation in speech perception is used in hearing aid devices. As might be expected given the success of the Tadoma method, tactile sensations delivered to the fingers improves the performance of speech perception in normal and hearing-impaired individuals (Auer et al. 1998; Cowan et al. 1990). Accordingly, there are devices designed for the hand. These devices provide speech information such as formants and amplitude using either or both electro-tactile 
Ito - Orofacial Curaneous Function

stimulation or vibro-tactile stimulation in conjunction with auditory information. Attempts have also been made to support speech perception via tactile devices alone (Galvin et al. 1999).

\section{Conclusions}

This chapter described the kinesthetic role of cutaneous afferents in orofacial motion and speech processing. Although the neural mechanisms and functions are not yet fully understood, the importance of facial cutaneous afferents in speech motor control and learning is clear because we accurately detect orofacial movements in spite of a lack of muscle proprioceptors in most perioral muscles. Specifically, orofacial cutaneous mechanoreceptors show a particular discharge pattern in response to facial motion, including motions involved in speaking. Accordingly, stretching the skin is an effective tool for investigating somatosensory function in speech processing. Studies using somatosensory modulation associated with facial skin deformation demonstrate the kinesthetic role of cutaneous afferents in speech motor control and learning. In particular, cutaneous mechanoreceptors are narrowly tuned at the skin lateral to the oral angles. In addition to their role in speech production, cutaneous afferents associated with articulatory motion also affect the perception of speech sounds. Speech sounds may possibly serve to tune the motor system, including kinesthetic processing, during language acquisition and vice versa. 
Ito - Orofacial Curaneous Function

\section{Further Reading}

Siemionow, Maria, Bahar B. Gharb \& Antonio Rampazzo. 2011. The face as a sensory organ. Plast Reconstr Surg 127.652-62. 
Ito - Orofacial Curaneous Function

\section{Reference}

Auer, Edward T., Jr., Lynne E. Bernstein \& David C. Coulter. 1998. Temporal and spatio-temporal vibrotactile displays for voice fundamental frequency: an initial evaluation of a new vibrotactile speech perception aid with normal-hearing and hearing-impaired individuals. J Acoust Soc Am 104.2477-89.

Barlow, Steven M. 1987. Mechanical frequency detection thresholds in the human face. Exp Neurol 96.253-61.

Bratzlavsky, Marc. 1979. Feedback control of human lip muscle. Exp Neurol 65.209-17.

Champoux, François, Douglas M Shiller \& Robert J Zatorre. 2011. Feel what you say: an auditory effect on somatosensory perception. PLoS One 6.e22829.

Cohen, Dan A. D., Michel J. Prud'homme \& John F. Kalaska. 1994. Tactile activity in primate primary somatosensory cortex during active arm movements: correlation with receptive field properties. J Neurophysiol 71.161-72.

Collins, David F. \& Arthur Prochazka. 1996. Movement illusions evoked by ensemble cutaneous input from the dorsum of the human hand. J Physiol 496 ( Pt 3).857-71.

Collins, David F., Kathryn M. Refshauge \& Simon C. Gandevia. 2000. Sensory integration in the perception of movements at the human metacarpophalangeal joint. J Physiol 529 Pt 2.505-15. 
Ito - Orofacial Curaneous Function

Collins, David F., Kathryn M. Refshauge, Gabrielle Todd \& Simon C. Gandevia. 2005. Cutaneous receptors contribute to kinesthesia at the index finger, elbow, and knee. J Neurophysiol 94.1699-706.

Connor, Nadin P. \& James H. Abbs. 1998. Movement-related skin strain associated with goal-oriented lip actions. Exp Brain Res 123.235-41.

Cooper, Sybil. 1953. Muscle spindles in the intrinsic muscles of the human tongue. J Physiol 122.193-202.

Cordo, Paul, Victor S. Gurfinkel, Leslie Bevan \& Graham K. Kerr. 1995. Proprioceptive consequences of tendon vibration during movement. J Neurophysiol 74.1675-88.

Cowan, Robert S, Peter J Blamey, Karyn L Galvin, Julia Z Sarant, Joseph I Alcántara \& Graeme M Clark. 1990. Perception of sentences, words, and speech features by profoundly hearing-impaired children using a multichannel electrotactile speech processor. J Acoust Soc Am 88.1374-84.

Di Francesco, G., Antonio Nardone \& Marco Schieppati. 1986. Inhibition of jaw-closing muscle activity by tactile air-jet stimulation of peri- and intra-oral sites in man. Arch Oral Biol 31.273-8.

Edin, Benoni. 2001. Cutaneous afferents provide information about knee joint movements in humans. J Physiol 531.289-97.

Edin, Benoni B. 1992. Quantitative analysis of static strain sensitivity in human mechanoreceptors from hairy skin. J Neurophysiol 67.1105-13. 
Ito - Orofacial Curaneous Function

Edin, Benoni B. \& James H. Abbs. 1991. Finger movement responses of cutaneous mechanoreceptors in the dorsal skin of the human hand. J Neurophysiol 65.65770.

Edin, Benoni B., Gregory K. Essick, Mats Trulsson \& Kurt A. Olsson. 1995. Receptor encoding of moving tactile stimuli in humans. I. Temporal pattern of discharge of individual low-threshold mechanoreceptors. J Neurosci 15.830-47.

Edin, Benoni B. \& Niclas Johansson. 1995. Skin strain patterns provide kinaesthetic information to the human central nervous system. J Physiol 487 ( Pt 1).243-51.

Eklund, Göran \& Karl-Erik Hagbarth. 1966. Normal variability of tonic vibration reflexes in man. Exp Neurol 16.80-92.

Eriksson, Per-Olof, Gill S. Butler-Browne \& Lars-Eric Thornell. 1994. Immunohistochemical characterization of human masseter muscle spindles. Muscle Nerve 17.31-41.

Essick, Gregory K. \& Benoni B. Edin. 1995. Receptor encoding of moving tactile stimuli in humans. II. The mean response of individual low-threshold mechanoreceptors to motion across the receptive field. J Neurosci 15.848-64.

Folkins, John W. \& Charles R. Larson. 1978. In search of a tonic vibration reflex in the human lip. Brain Res 151.409-12. 
Ito - Orofacial Curaneous Function

Fowler, Carol A. \& Dawn J. Dekle. 1991. Listening with eye and hand: cross-modal contributions to speech perception. J Exp Psychol Hum Percept Perform 17.81628.

Galvin, Karyn L., Peter J. Blamey, Michael Oerlemans, Robert S. Cowan \& Graeme M. Clark. 1999. Acquisition of a tactile-alone vocabulary by normally hearing users of the Tickle Talker. J Acoust Soc Am 106.1084-9.

Gandevia, Simon C. 1985. Illusory movements produced by electrical stimulation of lowthreshold muscle afferents from the hand. Brain 108 ( Pt 4).965-81.

Gick, Bryan \& Donald Derrick. 2009. Aero-tactile integration in speech perception. Nature 462.502-4.

Gomi, Hiroaki, Takayuki Ito, Emi Z. Murano \& Masaaki Honda. 2002. Compensatory articulation during bilabial fricative production by regulating muscle stiffness. J Phonetics 30.261-79.

Goodwin, Guy M., D. Ian McCloskey \& Peter B. C. Matthews. 1972. Proprioceptive illusions induced by muscle vibration: contribution by muscle spindles to perception? Science 175.1382-4.

Gracco, Vincent L. \& James H. Abbs. 1985. Dynamic Control of perioral system during speech: kinematic analysis of autogenic and nonautogenic sensorimotor processes. J. of Neurophysiology 54.418-32. 
Ito - Orofacial Curaneous Function

Grill, Stephen E. \& Mark Hallett. 1995. Velocity sensitivity of human muscle spindle afferents and slowly adapting type II cutaneous mechanoreceptors. J Physiol 489 ( Pt 2).593-602.

Halata, Zdenek \& Bryce L. Munger. 1983. The sensory innervation of primate facial skin. II. Vermilion border and mucosa of lip. Brain Res 286.81-107.

Ito, Takayuki \& Hiroaki Gomi. 2007. Cutaneous mechanoreceptors contribute to the generation of a cortical reflex in speech. Neuroreport 18.907-10.

Ito, Takayuki, Hiroaki Gomi \& Masaaki Honda. 2004. Dynamical simulation of speech cooperative articulation by muscle linkages. Biol Cybern 91.275-82.

Ito, Takayuki \& David J Ostry. 2010. Somatosensory contribution to motor learning due to facial skin deformation. J Neurophysiol 104.1230-8.

—. 2012. Speech sounds alter facial skin sensation. J Neurophysiol 107.442-7.

Ito, Takayuki, Mark Tiede \& David J Ostry. 2009. Somatosensory function in speech perception. Proc Natl Acad Sci U S A 106.1245-8.

Johansson, Roland S., Mats Trulsson, Kurt Â. Olsson \& James H. Abbs. 1988a. Mechanoreceptive afferent activity in the infraorbital nerve in man during speech and chewing movements. Exp Brain Res 72.209-14.

Johansson, Roland S., Mats Trulsson, Kurt Â. Olsson \& Karl-Gunnar Westberg. 1988b. Mechanoreceptor activity from the human face and oral mucosa. Exp Brain Res 72.204-8 
Ito - Orofacial Curaneous Function

Kubota, Kinziro \& Toshiaki Masegi. 1977. Muscle spindle supply to the human jaw muscle. J Dent Res 56.901-9.

Lamarre, Y. \& James P. Lund. 1975. Load compensation in human masseter muscles. J Physiol 253.21-35.

Lametti, Daniel R., Sazzad M. Nasir \& David J. Ostry. 2012. Sensory preference in speech production revealed by simultaneous alteration of auditory and somatosensory feedback. J Neurosci 32.9351-8.

Larson, Charles R., John W. Folkins, Michael D. McClean \& Eric M. Muller. 1978. Sensitivity of the human perioral reflex to parameters of mechanical stretch. Brain Res 146.159-64.

Lennartsson, Bertil. 1979. Muscle spindles in the human anterior digastric muscle. Acta Odontol Scand 37.329-33.

Lund, James P., Yves Lamarre, Gilles Lavigne \& G. Duquet. 1983. Human Jaw Reflexes, ed. by J.E. Desmedt, 739-55. New York: Raven Press.

Marsden, Charles D., Patrick A. Merton \& H. B. Morton. 1972. Servo action in human voluntary movement. Nature 238.140-3.

McClean, Michael D. \& John L. Clay. 1994. Evidence for suppression of lip muscle reflexes prior to speech. Exp Brain Res 97.541-4.

McClean, Michael D. \& Anne Smith. 1982. The reflex responses of single motor units in human lower lip muscles to mechanical stimulation. Brain Res 251.65-75. 
Ito - Orofacial Curaneous Function

McCloskey, Douglas I. 1978. Kinesthetic sensibility. Physiol Rev 58.763-820.

McGlone, Francis \& David Reilly. 2010. The cutaneous sensory system. Neurosci Biobehav Rev 34.148-59.

McGurk, Harry \& John MacDonald. 1976. Hearing lips and seeing voices. Nature 264.746-8.

Miles, Timothy S., Stanley C. Flavel \& Michael A. Nordstrom. 2004. Stretch reflexes in the human masticatory muscles: a brief review and a new functional role. Hum Mov Sci 23.337-49.

Munger, Bryce L. \& Zdenek Halata. 1983. The sensory innervation of primate facial skin. I. Hairy skin. Brain Res 286.45-80.

Murray, Gregory M. \& Iven J. Klineberg. 1984. Electromyographic recordings of human jaw-jerk reflex characteristics evoked under standardized conditions. Arch Oral Biol 29.537-49.

Nasir, S. M. \& D. J. Ostry. 2009. Auditory plasticity and speech motor learning. Proc Natl Acad Sci U S A 106.20470-5.

Nasir, Sazzad M. \& David J. Ostry. 2008. Speech motor learning in profoundly deaf adults. Nat Neurosci 11.1217-22.

Neilson, Peter D., Gavin Andrews, Barry E. Guitar \& Peter T. Quinn. 1979. Tonic stretch reflexes in lip, tongue and jaw muscles. Brain Res 178.311-27. 
Ito - Orofacial Curaneous Function

Nordin, Magnus \& Karl-Erik Hagbarth. 1989. Mechanoreceptive units in the human infra-orbital nerve. Acta Physiol Scand 135.149-61.

Nordin, Magnus \& Lars Thomander. 1989. Intrafascicular multi-unit recordings from the human infra-orbital nerve. Acta Physiol Scand 135.139-48.

O'Dwyer, Nicolas J., Peter T. Quinn, Barry E. Guitar, Gavin Andrews \& Peter D. Neilson. 1981. Procedures for verification of electrode placement in EMG studies of orofacial and mandibular muscles. J Speech Hear Res 24.273-88.

Okdeh, Atef M., Mervyn F. Lyons \& Samuel W. Cadden. 1999. The study of jaw reflexes evoked by electrical stimulation of the lip: the importance of stimulus intensity and polarity. J Oral Rehabil 26.479-87.

Ostry, David J., Paul L. Gribble, Mindy F. Levin \& Anatol G. Feldman. 1997. Phasic and tonic stretch reflexes in muscles with few muscle spindles: human jaw-opener muscles. Exp Brain Res 116.299-308.

Proske, Uwe \& Simon C Gandevia. 2009. The kinaesthetic senses. J Physiol 587.413946.

Prud'homme, Michel J., Dan A. D. Cohen \& John F. Kalaska. 1994. Tactile activity in primate primary somatosensory cortex during active arm movements: cytoarchitectonic distribution. J Neurophysiol 71.173-81. 
Ito - Orofacial Curaneous Function

Reed, Charlotte M., William M. Rabinowitz, Nathaniel I. Durlach, Louis D. Braida, Susan Conway-Fithian \& Martin C. Schultz. 1985. Research on the Tadoma method of speech communication. J Acoust Soc Am 77.247-57.

Sams, Mikko, Riikka Möttönen \& Toni Sihvonen. 2005. Seeing and hearing others and oneself talk. Brain Res Cogn Brain Res 23.429-35.

Shaiman, Susan \& Vincent L. Gracco. 2002. Task-specific sensorimotor interactions in speech production. Exp Brain Res 146.411-8.

Smith, Anne, Christopher A. Moore, David H. McFarland \& Christine M. Weber. 1985a. Reflex responses of human lip muscles to mechanical stimulation during speech. $\mathbf{J}$ Mot Behav 17.148-67.

Smith, Anne, Christpher A. Moore \& Carol A. Pratt. 1985b. Distribution of the human jaw stretch reflex response elicited by percutaneous, localized stretch of jawclosing muscles. Exp Neurol 88.544-61.

Stål, Per, Per-Olof Eriksson, Anders Eriksson \& Lars-Eric Thornell. 1987. Enzymehistochemical differences in fibre-type between the human major and minor zygomatic and the first dorsal interosseus muscles. Arch Oral Biol 32.833-41.

—. 1990. Enzyme-histochemical and morphological characteristics of muscle fibre types in the human buccinator and orbicularis oris. Arch Oral Biol 35.449-58.

Sumby, W. H. \& Irwin Pollack. 1954. Visual Contribution to Speech Intelligibility in Noise. J Acoust Soc Am 26.212-15. 
Ito - Orofacial Curaneous Function

Takano, Sayoko \& Kiyoshi Honda. 2007. An MRI analysis of the extrinsic tongue muscles during vowel production. Speech Communication 49.49-58.

Tremblay, Stéphanie, Guillaume Houle \& David J. Ostry. 2008. Specificity of speech motor learning. J Neurosci 28.2426-34.

Tremblay, Stéphanie, Douglas M. Shiller \& David J. Ostry. 2003. Somatosensory basis of speech production. Nature 423.866-69.

Trulsson, Mats \& Roland S. Johansson. 2002. Orofacial mechanoreceptors in humans: encoding characteristics and responses during natural orofacial behaviors. Behav Brain Res 135.27-33.

Vatikiotis-Bateson, Eric., Takaaki Kuratate, Myuki Kamachi \& Hani Yehia. 1999. Facial deformation parameters for audiovisual synthesis. Paper presented to the Auditory-Visual Speech Processing, Santa Cruz, CA, USA, 1999.

Weinstein, Sidney. 1968. Intensive and extensive aspects of tactile sensitivity as function of body part, sex and laterality. The Skin Senses, ed. by K. DR, 195-222. Springfield IL: Thomas.

Yehia, Hani, Philip Rubin \& Eric Vatikiotis-Bateson. 1998. Quantitative association of vocal-tract and facial behavior. Speech Communication 26.23-43. 
Ito - Orofacial Curaneous Function

Keywords: Orofacial skin sensation, Facial proprioception, Speech production, Motor adaptation, Speech learning, Somatosensory processing, Somatosensory-auditory integration.

Short biographical note: Takayuki Ito has been senior research scientist in Haskins Laboratories since 2005 and Adjunct Professor in University of Connecticut since 2011. He earned his $\mathrm{PhD}$ at Chiba University, Japan in 1999. His research interest is somatosensory processing in speech production and perception. 